\title{
5 Research Square

\section{Circular RNA profiling of buffalo myoblasts reveals an abundant circPICALM that promotes myoblast differentiation}

\section{Mingming Song}

Guangxi University

Mengjie Chen

Guangxi University

Kongwei Huang

Guangxi University

Dandan Zhong

Guangxi University

Yaling Chen

Guangxi University

Jiayuan Fu

Guangxi University

Mingsheng Jiang

Guangxi University

Qingyou Liu

Guangxi University

Deshun Shi

Guangxi University

Hui Li (D lihui3876@163.com )

Guangxi University https://orcid.org/0000-0002-5604-0592

\section{Research}

Keywords: Buffalo, RNA-seq, CircRNA, Myogenesis

Posted Date: May 11th, 2020

DOI: https://doi.org/10.21203/rs.3.rs-23175/v1

License: (1) (1) This work is licensed under a Creative Commons Attribution 4.0 International License.

Read Full License 


\section{Abstract}

Background Muscle development is a precisely orchestrated and complex process, and circular RNAs (circRNAs) has been demonstrated to play important roles in skeletal muscle growth and development. However, the regulatory functions of circRNA during buffalo muscle developmental processes have not been understood.

Results In this study, Ribo-Zero RNA-Seq was performed to investigate the circRNAs expression profiles of proliferated and differentiated buffalo myoblasts. A stringent set of 3,142 circRNAs was finally characterized. Comparing the expression profiles of circRNAs revealed that 110 circRNAs were expressed differentially during myoblast differentiation. We focused on the role of a candidate circRNA, which was named circPICALM based on its host gene PICALM, and was highly (but differentially) expressed in proliferated and differentiated myoblasts. Flow cytometry, EdU incorporation, and Western blotting assays demonstrate that circPICALM promoted myoblasts proliferation and inhibited cells apoptosis. Moreover, overexpression of circPICALM promoted the differentiation of primary buffalo myoblasts. Moreover, circPICALM in vivo stimulated skeletal muscle regeneration in cardiotoxin-induced muscle injury. The RNA pulldown results showed that circPICALM could capture TUBA1B protein, revealing that circPICALM might exert its biological function by binding TUBA1B protein.

Conclusions These results demonstrate that the novel non-coding regulator circPICALM induces myoblast differentiation and skeletal muscle regeneration.

\section{Introduction}

Myogenesis is initiated in the embryonic mesoderm and is a highly orchestrated biological process (Margaret, 2006)\{Buckingham, 2006 \#1;Buckingham, 2006 \#1\}. After the muscle stem cells differentiate into myoblasts, they further form multinucleated myotubes through proliferation, differentiation and fusion, thereby finally forming mature muscle fibers (Margaret et al., 2003). In addition, cell apoptosis also occurs at these stages, which ensures the equilibrium of muscle development ( $P$ et al., 2001; Agnieszka et al., 2015). The growth and development of skeletal muscle are regulated by a series of transcription factors, including myogenic regulatory factors (MRFs) family (Thomas and Mathias, 2011; Manuel et al., 2017), paired box protein 3/7 (Pax3/7) and myocyte enhancer factor 2 (MEF2) family ( and E, 1999; A and A, 2000; Margaret et al., 2003). Moreover, these transcription factors are controlled by protein-coding genes and non-coding genes directly or indirectly. Although the origin of skeletal muscle and the level of genetic hierarchy that determines its formation are well clearly, the regulatory mechanisms involved in this process are not well understood. Therefore, discovery of new regulatory factors and an improved understanding of molecular mechanisms of skeletal muscle development remain urgent needs.

Non-coding RNA, for example, circular RNA (circRNA) has been reported to be involved in myogenic gene regulatory network (A et al., 1999; Simona et al., 2018). In the last decade, Genomic analysis of 
mammalian transcriptomes indicates that over $50 \%$ of transcripts are not translated into proteins but as functional RNAs or junk, including microRNA (miRNA), long noncoding RNA (IncRNA), and circRNA. CircRNA was first found as a viroid in 1976 (Sanger et al., 1976), and was originally thought to be caused by gene splicing error ( $T$ and $M, 1979)$. Due to the rapid development of bioinformatics and RNA sequencing (RNA-seq) technologies, a large number of circRNAs have been identified in different species. CircRNA plays an indispensable role in regulating gene activity at the transcription and post-transcription levels, such as regulating RNA-protein interactions and alternative splicing, acting as a molecular scaffold in protein-DNA complexes, and acting as a microRNA sponge (B et al., 2013; Sebastian et al., 2013; Yang et al., 2013; Zhaoyong et al., 2015). In addition, in mammalian brains and cancer cells, circRNA expression is often abnormally expressed, and some circRNAs have been reported to be associated with human neurodegenerative diseases and cancer progression (Lukiw, 2013; Agnieszka et al., 2015; M and Jeroen, 2017).

In fact, various non-coding RNAs have been found in muscle tissue and have been shown to regulate muscle growth and development. LncRNA is involved in the transcription/epigenetic regulation of chromatin by acting as molecular scaffold or decoy to activate or inhibit transcription, thereby regulating myoblast differentiation (Yuying et al., 2018). CircRNA has been identified in muscles of humans, mice, and pigs, and has been found to affect muscle function (Ivano et al., 2017; Jianning et al., 2018; Wei et al., 2018). CircLM07, circFUT10 and circFGFR4 have been shown as competing endogenous RNAs (ceRNAs) to bind miRNA and regulate bovine myoblast differentiation (Xuefeng et al., 2017; Hui et al., 2018a; Hui et al., 2018b).

The Chinese buffalo was originally used for labor purposes, and is now mainly selected for meat or milk production (Jieping et al., 2019). Compared with beef, buffalo meat has less fat and cholesterol, which means that buffalo is healthier and can bring significant cardiovascular benefits. Our previous research showed that under the same feeding and management methods, the quality of buffalo meat can be comparable to beef, but there are significant differences in shear force and muscle fiber structure (Hui et al., 2020). To further analyze the regulatory role of non-coding RNA in the development of buffalo muscles, we used the Ribo-Zero RNA-seq method to characterize the circRNA expression profile during the proliferation and differentiation stages of buffalo myoblasts. In the second part of our study, we focused on circPICALM as a candidate circRNA, which was differentially and highly expressed in buffalo myoblasts, and explored its role in cell proliferation and differentiation. These evidences not only provide genetic resources for understanding muscle development, but also reveal new insights into circRNAs that have functional roles in myogenesis and muscle regeneration.

\section{Methods}

\section{Sample preparation}

Embryo and adult buffalo tissues (heart, spleen, lung, liver, brain, kidney, leg muscles and long muscles) were collected and quickly frozen in liquid nitrogen. Animal health and research programs have been 
approved by the Animal Health Committee of the School of Animal Science and Technology of Guangxi University.

\section{RNA-seq library construction and sequencing analysis}

We electrophoresed RNA extracts on denatured agarose gels to assess total RNA yield, and then used a NanoDrop spectrophotometer (NanoDrop, Wlinington, USA) and an Agilent 2100 bioanalyzer (Agilent, Santa Clara, CA, United States) for quantification of RNA extracts. Ribosomal RNA was removed (to obtain sequence information for linear transcripts), and Ribo-Zero ${ }^{\text {TM }}$ rRNA Removal Kit (Epicentre, Madison, Wisconsin, USA) was used before constructing the RNA-seq library. Library preparation and Illumina sequencing analysis have been described in previous studies (Bing et al., 2015).

\section{Functional analysis of GO and KEGG pathway analysis}

Gene ontology (GO) analysis (http://www.geneontology.org) for characterizing circRNA host genes. GO terminology provides information about biological processes (cellular components or metabolic pathways) involving genes and highlights molecular functions while reducing complexity. In order to gain insight into the molecular interaction and response network of circRNAs that have been found to be differentially expressed using visualization and integration (Version 6.7; http://david.ncifcrf.gov), we also conducted the Kyoto Protocol Encyclopedia of Genes and Genomes (KEGG; http://www.kegg.jp) pathway analysis. In both cases, significant enrichment of a given GO term or pathway between entities is indicated by $-\log _{10}$ P-values for up and down-regulation.

\section{RNA isolation, cDNA synthesis, and real-time qPCR}

Extract total RNA from cells and tissues using Trizol reagent (TaKaRa, Dalian, China). Removal of genomic DNA (TaKaRa, Dalian, China) and reverse transcription of total RNA using PrimeScript ${ }^{\text {TM }}$ RT kit with gDNA eraser. Real-time quantitative PCR (qPCR) was performed three times on the Bio-Rad CF96 system (Bio-Rad, USA) using SYBR green kit (Genestar, Beijing, China). For RNase R treatment, incubate 1 $\mu \mathrm{g}$ of total RNA at $37^{\circ} \mathrm{C}$ for 20 minutes with or without 2 units of $1 \mu \mathrm{g}$ RNase $\mathrm{R}$, and purify according to the RNeasy MinElute cleaning kit (Qiagen, Hilden, Germany). The primers are listed in Table S1.

\section{Plasmid construction}

The spliced full-length circPICALM (Text S1) was inserted into the pCD2.1-ciR vector (Geneseed, Guangzhou, China) to construct its expression plasmid (pcD-circPICALM). The primers are listed in Table S1.

\section{Cell culture and treatments}

Perform the myoblast isolation step as previously described (Xuefeng et al., 2017). The brief steps related to cells separation are as follows description. The muscle tissue was digested with collagenase at $37^{\circ} \mathrm{C}$ water bath about one hour. Then, the digested muscle tissue was centrifuged at a low speed, and the 
supernatant was discarded, and the remaining tissue was filtered through a 100-mesh filter. The filtrate was washed three times with PBS, and finally resuspended in growth medium containing $20 \%$ FBS and $1 \%$ penicillin/streptomycin to resuspend the cells. Then, the cells were seeded at a certain density on the cell culture plate at $37^{\circ} \mathrm{C}$ in $5 \% \mathrm{CO}_{2}$. To induce cell differentiation, the growth medium is replaced with a differentiation medium containing $2 \%$ horse serum, when the cells grow to contact adhesion.

\section{Fluorescence in situ hybridization}

Perform fluorescence in situ hybridization (FISH), following the instructions of the manufacturer of the in situ hybridization reagent (Servicebio, Wuhan, China). Briefly, buffalo myoblasts were cultured on glass slides and treated with $4 \%$ paraformaldehyde solution for 20 minutes. Incubate fixed cells with premixed solution at $37^{\circ} \mathrm{C}$ for 1 hour. Then, the fixed cells were incubated with the hybridization solution containing circPICALM probe. After the overnight incubation, the cells were washed multiple times, and then incubated in the dark for 8 minutes after adding the DAPI solution to the cell surface. Finally, observe these cells by using a fluorescence microscope (Nikon Japan).

\section{CCK-8 and EdU assay}

The proliferation status of buffalo myoblasts was studied using CCK-8 (Tiandz, Beijing, China) and CellLight $^{\text {TM }}$ EdUApollo® 567 in vitro imaging kit (RiboBio). For detailed steps, refer to the manufacturer's instructions.

\section{Flow cytometry}

Cell cycle and apoptosis detection were performed by flow cytometry (Becton Dickinson, FACSCalibur, USA). To analyze the cell cycle, use the cell cycle test kit (Multisciences, Hangzhou, China). For detailed steps, refer to the manufacturer's instructions.

\section{Western blotting analysis}

To extract cellular proteins, RIPA buffer containing 1\% PMSF (Solarbio, Beijing, China) was used. To determine protein concentration, use BCA kit (Beyotime, Shanghai, China). The separated proteins were subjected to $12 \%$ SDS-polyacrylamide gel electrophoresis and transferred to polyvinylidene fluoride membranes. Combine the membrane with anti-CyclinD1 (Wanli Biou, Shenyang, China), anti-PCNA (Wanli Biou, Shenyang, China), anti-MyHC (GeneTex, USA) and $\beta$-actin (Abcam, Cambridge, England) and secondary antibodies, and then use ECL Plus (Solarbio, Beijing, China) was exposed and quantified using the ChemiDoc XRS + system (Bio-Rad, USA).

\section{Cellular immunofluorescence analysis}

Absorb and discard the culture solution in the cell culture dish, fix with $4 \%$ cold paraformaldehyde for 20 minutes, then wash with PBS 3 times for 5 minutes each time, then use $0.5 \%$ Triton x-osmosis Cells were washed for 10 minutes and 10 minutes with PBS. Seal with $5 \%$ bovine serum albumin (BSA) for 30 
minutes and wash 3 times with PBS. Anti-MyHC (GeneTex, USA) was incubated overnight on a shaker and washed three times with PBS. The fluorescent-labeled secondary antibody was incubated at room temperature in the dark for 2 hours, and then washed 4 times with PBS. The nuclei were stained with DAPI for 10 minutes, then 3 times with PBS, and observed under a fluorescent microscope (Nikon Japan).

\section{RNA pull-down assay}

The detailed RNA pulldown assay procedure is as described above (B et al., 2002; Tasneem et al., 2006). The complete sequence fragment of circPICALM gene was synthesized and cloned into pBluescript II SK

(+) to prepare plasmid template. This template was used to prepare biotinylated RNA probes. The sample was separated by magnetic beads and the pull-down protein mixture for mass spectrometry analysis (Sangon Biotech, Shanghai, China).

\section{Animal research}

C57BL/6 strain mice were purchased from Guangxi Medical University (Nanning, China). The research protocol and animal care were approved by the Animal Care Committee of the College of Veterinary Medicine of Guangxi University. To induce skeletal muscle damage, Cardiotoxin (CTX; Whiga) was injected into the anterior tibial muscle of approximately 5 weeks old mice as a $50 \mu \mathrm{L} \mathrm{CTX}$ solution (10 $\mu \mathrm{M})$. In each treatment group, the expression plasmid pcD-circPICALM (Engreen Biosystem Co., Ltd.) with Entranster $^{\mathrm{TM}}$ in vivo transfection reagent was injected into the tibialis anterior muscle of 3 mice. The injection time after CTX treatment was 12, 48 and 96 hours. Three other mice were injected with glucose solution without plasmids as a control group. After CTX treatment (144 hours), the tibialis anterior muscle was collected for RNA extraction and detected by qPCR.

\section{Frozen sections and HE staining}

The collected tibialis anterior muscle tissue of mice was fixed with $4 \%$ paraformaldehyde for 24 hours according to standard procedures. Three slices were taken from each of the three mice, and $5 \mathrm{~mm}$ thick slices were embedded in resin. Then, after HE staining, it was sealed with resin and observed under a fluorescent microscope (Nikon Japan).

\section{Statistical analyses}

Data were presented as the mean \pm SEM. $P$-values were calculated by a two-tailed Student's t-test. $P<$ 0.05 was considered of significant difference.

\section{Results}

\section{Expression profiles of circRNAs in proliferating and differentiating buffalo myoblasts}

A large number of RNAs were identified in proliferating and differentiating buffalo myoblasts by using Ribo-Zero RNA-Seq (Table 1). After removing ribosomal RNA (rRNA ${ }^{-}$), we identified 3,142 circRNAs 
candidates (Table 2, Additional file 1), and 77,045,729 77,493,289 and 75,896,937 81,224,612 unique mapped reads were obtained from the proliferating and differentiating myoblasts libraries, respectively (Table 1). We found that the genomic loci of circRNAs are widely distributed on the entire chromosome (Figure 1a), and longer chromosomes produce more reads, which suggests that the number of circRNAs produced in the chromosome increases with the length of the chromosome. In order to fully identify circRNAs with potential functions during the proliferation and differentiation of buffalo myoblasts, the number of circRNAs was counted. The results showed that 1,118 and 1,063 circRNAs were specific to the proliferating and differentiating libraries, respectively (Figure 1b). Moreover, about $96.93 \%$ circRNAs are derived from exons, and about $1.33 \%$ are derived from introns, but only a small number of circRNAs are derived from intergenic regions (Figure 1c).

\section{Differentially expressed circular RNAs}

From the fold change and the adjusted level of significance, the differential circRNAs were screened and found that 63 circRNAs were down-regulated and 47 circRNAs were up-regulated when comparing proliferating myoblasts to differentiating cells (Figure 2a, Additional file 2). To determine whether the expression of circRNA is affected by host genes, we directly compared the up-regulated and downregulated expression of circRNA $(n=110)$ and its corresponding mRNA $(n=85)$ expression changes between proliferating and differentiated myoblast cells. The expression patterns of many circRNAs at different developmental stages are different from their maternal genes, and the changes in mRNA expression do not fully explain the observed circRNA expression, indicating that circRNAs are not random by-products of canonical splicing (Figure 2b).

In addition, we used GO and KEGG to analyze differentially expressed circRNA and the signal transduction pathways involved (Figure 2c-e, Additional file 3). To further analyze the possible function of circRNA, a cluster heat map of differentially expressed circRNA was prepared (Figure 2f), which shows the abundance of the maternal gene transcripts of the top 100 circRNAs with the largest differences (Figure 2g). Moreover, the expression level of differentially expressed circRNA (mean SRPBM $=28.63$ ) is consistent with its host gene (mean FPKM = 39.66) (Additional file 2 and Additional file 4).

\section{Characteristics of circRNA in buffalo myoblasts}

By analyzing the characteristics of circRNA in buffalo myoblasts, we found that most circRNAs consist of 2 to 6 exons (Figure 3a), and a parent gene can produce 1 4 circRNAs, and even more than 10 circRNAs (Figure 3b). Most of circRNAs have a length of $>10,000$ nucleotides (nt), with an average length of 17,474 nt including flanking introns lengths (Figure 3c, Table 2). Moreover, the expression of circRNAs in buffalo myoblasts during proliferation and differentiation are below 50 (SRPBM, Figure 3d).

\section{Competing endogenous RNA network in buffalo myoblasts}

CircRNAs and target genes bind to miRNAs competitively, thereby removing the inhibitory effect of miRNAs on target gene expression. In order to build a ceRNA network, we selected all the differentially 
expressed circRNAs and predicted their binding miRNAs based on bioinformatics software. Most of these selected miRNAs are related to muscle development. Finally, we constructed a buffalo myoblast miRNAcircRNA network (Figure S1).

\section{The expression of circPICALM in buffalo muscle development}

In our sequencing data, we found that circPICALM was differentially expressed in buffalo proliferated and differentiated myoblasts, and was specifically expressed in adult buffalo skeletal muscle (Figure $4 \mathrm{~b}$ and c, Additional file 2). According to this characteristic, divergent primers and convergent primers were designed to use buffalo cDNA and genomic DNA as templates for PCR. CircPICALM was found to be amplified only in cDNA sample (Figure 4a). Tissue expression assay revealed that circPICALM was highly expressed in fetal and adult skeletal muscle (Figure $4 \mathrm{~b}$ and $\mathrm{c}$ ). Furthermore, the expression of circPICALM in myoblasts in the proliferation phase was higher than that in the differentiation phase (Figure 4d), suggesting potential function of circPICALM in buffalo muscle development. RNase R digestion assay further confirmed that circPICALM was circular in form (Figure 4e). Therefore, we selected circPICALM as a candidate circRNA, which is transcribed from the PICALM gene on chromosome with a full length of 347 nucleotides (Text S1).

\section{Effects of circPICALM on cell proliferation and cell apoptosis}

To clarify the effect of circPICALM on cell proliferation and apoptosis, the circPICALM was overexpressed using plasmid pCD2.1 transfecting into buffalo myoblasts (Figure 5a). We found pCD2.1-circPICALM promoted the expression of CyclinD1 at mRNA levels (Figure 5b). EdU staining analysis showed that circPICALM increased the proportion of EdU positive cells, indicating that circPICALM has a promoting effect on cell proliferation (Figure 5c). Similarly, cell cycle analysis showed that circPICALM increased the proportion of myoblasts in S and G2 phases and decreased the number of cells in G0/G1 phases (Figure $5 d)$. These results suggest that circPICALM promotes buffalo myoblasts proliferation.

To analyze whether circPICALM affects myoblast apoptosis, qPCR, Western blot and Annexin VFITC/propidium iodide (PI) staining analysis were performed. Bcl-2, Bax, and p53 have been considered as key regulators of apoptosis, and we asked whether they are regulated by circPICALM to influence myoblast apoptosis. We found circPICALM to decrease the expression of p53 and Bax, and increase the expression of Bcl-2 at mRNA and protein levels (Figures 6a and b). Moreover, the Annexin V-FITC/PI staining assay demonstrated that circPICALM overexpression could inhibit cell apoptosis lightly, but not significantly $(P>0.05$; Figure $6 \mathrm{c})$. These results reveal that circPICALM inhibits cell apoptosis in buffalo myoblasts.

\section{CircPICALM promotes myoblasts differentiation}

In order to determine whether circPICALM has a regulatory effect on cell differentiation, we examined the expression of MyoD and MyHC in primary buffalo myoblasts treated with pCD-circPICALM. It was found that circPICALM overexpression markedly promoted MyHC expression at the mRNA and protein levels 
(Figure 7a and b). Immunofluorescence analysis showed that circPICALM significantly promoted MyHC expression and induced myotube formation (Figure 7c). These results show that circPICALM plays a role in promoting the differentiation of myoblasts.

\section{CircPICALM promotes skeletal muscle regeneration}

To further validate the role of circPICALM in skeletal muscle development, we conducted in vivo experiments using C57BL/6 mice injected with cardiotoxin (CTX) to induce mice muscle damage. Experimentally increased the expression of circPICALM by transfecting circPICALM overexpression plasmid, and we found that the expression level of circPICALM was considerably induced after the skeletal muscle injury (Figure 8a and c). The mRNA levels of Pax3 and Pax7 which increase proliferative rate and prevents precocious myogenic differentiation (Collins et al., 2018), also increased the third day (Figure 8b), but reduced to the same level as the control group on day 7 (Figure 8d). Hematoxylin and eosin (HE) staining analysis revealed that the muscle fibers in the circPICALM group were substantially repaired, and the numbers of muscle fibers were more than in the control group (Figure 8 e). These results suggest that circPICALM stimulates skeletal muscle regeneration in vivo.

\section{CircPICALM directly binds the NPM1,TUBA1B and PLEC proteins}

According to the subcellular localization, circRNAs play different regulatory roles: circRNAs in the cytoplasm could bind microRNAs or protein to perform post-transcriptional regulation, but circular RNAs in the nucleus may play a regulatory role by affecting gene transcription. In this study, FISH assays were performed to detect the cellular localization of circPICALM, and the results suggested that circPICALM preferentially distribute in the cytoplasm (Figure 9a). Considering that circPICALM is mainly located in the cytoplasm, we hypothesize that circPICALM may bind RNA or protein to regulate myoblast differentiation. The RNA pulldown result indicated that circPICALM can capture NPM1, TUBA1B and PLEC proteins (Table 3, Figure 9b). Studies have shown that TUBA1B knockout in hepatocellular carcinoma cells inhibits cell proliferation (Lu et al., 2013), revealing that circPICALM may exert its biological function by binding TUBA1B protein. Therefore, modulation the expression of circPICALM may emerge as an effective breeding strategy attempting to alter muscle mass in buffalo.

\section{Discussion}

Our current study was the first to analyze and annotate the type and relative abundance of circRNA in buffalo myoblasts using high-throughput RNA-seq. We characterized a novel circRNA, circPICALM, involved in skeletal muscle development based on data from transcriptome sequencing. Our in vitro experiments suggested that circPICALM promotes myogenesis and myotube formation in buffalo myoblasts. Consistently, in vivo experiments have shown that overexpression of circPICALM induces muscle regeneration.

CircRNAs have been reported to be involved in the regulation of gene expression during skeletal muscle growth and development (Reut et al., 2014). Depending on its location in the cell, circRNAs usually have 
multiple regulatory mechanisms. In the nucleus, circRNA can serve as a molecular scaffold to recruit transcription factors or transcriptases to mediate gene transcription (Zhaoyong et al., 2015). In the cytoplasm, circRNA could compete with the target gene to bind miRNA to reduce the miRNA's inhibitory effect on the target gene (B et al., 2013; Sebastian et al., 2013). For example, circFUT10 and circFGFR4 alleviate their inhibitory effects on Wnt3a and SRF by combining miR-133a and miR-107, respectively, to regulate myogenesis (Hui et al., 2018a; Hui et al., 2018b). In this study, using high-throughput RNA-seq analysis, 1,118 and 1,063 circRNAs were identified in proliferating and differentiating buffalo myoblasts, respectively. The differential circRNAs were screened and found that 63 circRNAs were downregulated and 47 circRNAs were up-regulated when comparing proliferating myoblasts to differentiating cells. We identified a circRNA transcript circPICALM and found that its expression was highly in skeletal muscle. Moreover, circPICALM was mainly localized in the cytoplasm. Combining of RNA pulldown assay, we found that circPICALM could bind TUBA1B protein to regulate the myogenesis of buffalo myoblasts.

Mostly, skeletal muscle dysplasia leads to muscle disease, for example muscle hypertrophy, atrophy and primary muscle disorders (Kavitha and Shankar, 2020). Noncoding RNAs have been proposed to play important roles in muscle regeneration and diseases. A panel of microRNAs including miR-1, $-133,-206$, -208 and -499 that may be molecular markers to diagnose diseases, as well as analyzing various myoblast types, including skeletal muscle, cardiac muscle and vascular smooth muscles (miR-1, -133a, -145, -208, -499) (Jian-Fu et al., 2006; Kyun et al., 2006; Eva et al., 2009; Jian-Fu et al., 2010). Similar to miR-133a, miR-23a was found to inhibit myoblast differentiation, revealing its potential role in treating muscular diseases. Notably, there is now accumulating evidence that circRNAs can change in their expression to regulate multiple biological responses related to the development of disease. For example, circZNF609 was detected in duchenne muscular dystrophy (DMD) muscle tissues, and controls cell proliferation and can be translated to encode a protein in DMD myoblasts (Ivano et al., 2017). In this report, we identified a circRNA which was up-regulated in differentiated myoblasts and induced myotubule formation. Importantly, overexpression of circPICALM promotes muscle regeneration in vivo, revealing that circPICALM has a potential role in muscular disease. Overall, investigating multiple ncRNAs is possible to develop treatments for muscle diseases in the future.

\section{Conclusions}

This study is the first to map the expression of circRNA in buffalo skeletal muscle. When comparing buffalo myoblasts in the proliferation phase and the differentiation phase, thousands of circRNAs were identified, and several of them showed very different abundances. Our research may lay a foundation for further research on the role of these circRNAs in the process of buffalo myogenesis. We characterized and evaluated the function of circPICALM, one of the differentially expressed circRNAs. Experimental results show that circPICALM promotes myoblast differentiation and inhibits apoptosis. Our research has increased the understanding of the genetic mechanism of buffalo skeletal muscle formation and may provide new molecular target for the precise breeding of buffalo for meat purpose in China. 


\section{Abbreviations}

circRNAs: circular RNAs; miRNA: microRNA; IncRNA: long non-coding RNA; ceRNAs: competing endogenous RNAs; GO: Gene ontology; KEGG: Kyoto Protocol Encyclopedia of Genes and Genomes; HE staining: hematoxylin-eosin staining; DMD: duchenne muscular dystrophy; FISH: fluorescence in situ hybridization; qPCR: real-time quantitative PCR; CTX: cardiotoxin.

\section{Declarations}

\section{Acknowledgements}

The authors would like to acknowledge Huaxu buffalo breeding farm for providing the buffalo.

\section{Funding}

Acknowledgements: This work was supported by the Guangxi Natural Science Foundation (Grant No. 2018GXNSFAA050086), Science and Technology Major Project of Guangxi (Grant No. AA17204051), and Innovation Project of Guangxi Graduate Education (Grant No. YCSW2019027).

\section{Availability of data and materials}

Not applicable.

\section{Authors' contributions}

Mingming Song gathered samples, Hui Li conceived the report, Qingyou Liu, Mingsheng Jiang and Deshun Shi participated in its design, Mingming Song and Mengjie Chen performed data analysis, Hui Li and Mingming Song interpreted results and drafted the manuscript, Kongwei Huang, Yaling Chen, Dandan Zhong and Jiayuan Fu participated in intellectual discussion. All authors have read and approved the manuscript, and ensure that this is the case.

\section{Ethics approval and consent to participate}

All experimental buffaloes were provided by Huaxu buffalo Breeding Farm, Nanning, Guangxi Zhuang Autonomous Region, China. The buffaloes were maintained according to the No. 5 Proclamation of the Ministry of Agriculture, P. R. China, and we confirm that all animal protocols and methods were approved by the Review Committee for the Use of Animal Subjects of Guangxi University.

\section{Consent for publication}

Not applicable.

\section{Conflicts of interest}

The authors declare they have no conflicts of interest. 


\section{References}

A, S., T. Y, W. T, I. M, N. I, and M. M. 1999. Circular dystrophin RNAs consisting of exons that were skipped by alternative splicing. \%J Human molecular genetics. 8(3)

A, S. L., and R. M. A. 2000. The molecular regulation of myogenesis. \%J Clinical genetics. 57(1)

Agnieszka, R.-W., S. Christin, G. Petar, J. Marvin, P. Natalia, G. Sebastian, H. Mor, B. Mikaela, B. Osnat, A.-F. Reut, H. Margareta, S. Luisa, P. Panagiotis, I. Andranik, Ö. Marie, R. Damian, K. Sebastian, and R. Nikolaus. 2015. Circular RNAs in the Mammalian Brain Are Highly Abundant, Conserved, and Dynamically Expressed. \%J Molecular cell. 58(5)

B, H. T., J. T. I, C. B. H, B. J. B, F. Bente, D. C. K, and K. Jørgen. 2013. Natural RNA circles function as efficient microRNA sponges. \%J Nature. 495(7441)

B, Y. B., V. D. C, V. J. P, M. R. K, T. A. M, G. K. M, C.-K. M. F, F. Henry, W. M. C. J, W. J. A, and L. P. J. 2002. Novel binding of HuR and poly(C)-binding protein to a conserved UC-rich motif within the 3 '-untranslated region of the androgen receptor messenger RNA. \%J The Journal of biological chemistry. 277(30)

Bing, G., G. P. L, C. L. M, Z. Guanghong, Z. Wangang, and D. B. P. 2015. Transcriptome analysis of cattle muscle identifies potential markers for skeletal muscle growth rate and major cell types. \%J BMC genomics. 16

Collins, C. A., V. F. Gnocchi, R. B. White, L. Boldrin, A. Perez-Ruiz, F. Relaix, J. E. Morgan, and P. S. Zammit. 2018. Integrated functions of $\mathrm{Pax} 3$ and $\mathrm{Pax} 7$ in the regulation of proliferation, cell size and myogenic differentiation. \%J PLoS ONE. 4(2)

Eva, v. R., Q. Daniel, J. B. A, S. L. B, Q. Xiaoxia, R. J. A, K. R. J, and O. E. N. 2009. A family of microRNAs encoded by myosin genes governs myosin expression and muscle performance. \%J Developmental cell. $17(5)$

Hui, L., Y. Jiameng, W. Xuefeng, S. Chengchuang, D. Dong, H. Yongzhen, L. Xianyong, P. Martin, L. Chuzhao, M. Yun, Q. Xinglei, B. Yueyu, and C. Hong. 2018a. CircFUT10 reduces proliferation and facilitates differentiation of myoblasts by sponging miR-133a. \%J Journal of cellular physiology. 233(6)

Hui, L., H. Kongwei, W. Pengcheng, F. Tong, S. Deshun, C. Kuiqing, L. Chan, S. Laiba, Q. Qian, R. Jue, and L. Qingyou. 2020. Comparison of Long Non-Coding RNA Expression Profiles of Cattle and Buffalo Differing in Muscle Characteristics. \%J Frontiers in genetics. 11

Hui, L., W. Xuefeng, Y. Jiameng, D. Dong, H. Dan, H. Yongzhen, L. Xianyong, P. Martin, L. Chuzhao, M. Yun, L. Fengpeng, B. Yueyu, and C. Hong. 2018b. circFGFR4 Promotes Differentiation of Myoblasts via Binding miR-107 to Relieve Its Inhibition of Wnt3a. \%J Molecular therapy. Nucleic acids. 11 
Ivano, L., D. T. Gaia, R. Francesca, M. Mariangela, B. Francesca, S. Olga, F. Alessandro, S. Tiziana, A. Adrian, W. Mark, L. Pietro, R. Nikolaus, and B. Irene. 2017. Circ-ZNF609 Is a Circular RNA that Can Be Translated and Functions in Myogenesis. \%J Molecular cell. 66(1)

J, N. F., and O. E. 1999. MEF2: a transcriptional target for signaling pathways controlling skeletal muscle growth and differentiation. \%J Current opinion in cell biology. 11(6)

Jian-Fu, C., M. E. M, T. J. Michael, W. Qiulian, C. T. E, H. S. M, C. F. L, and W. Da-Zhi. 2006. The role of microRNA-1 and microRNA-133 in skeletal muscle proliferation and differentiation. \%J Nature genetics. $38(2)$

Jian-Fu, C., T. Yazhong, L. Juan, D. Zhongliang, Y. Zhen, X. Xiao, and W. Da-Zhi. 2010. microRNA-1 and microRNA-206 regulate skeletal muscle satellite cell proliferation and differentiation by repressing Pax7. $\% \mathrm{~J}$ The Journal of cell biology. 190(5)

Jianning, C., Z. Qin, L. Daojun, W. Yingying, R. M. Ali, C. Yan, L. Peilin, X. Xiaoyu, X. Huaming, W. Anxiang, Z. Li, T. Guoqing, L. Mingzhou, J. Anan, L. Yihui, F. Yuhua, J. Yanzhi, and L. Xuewei. 2018. Comprehensive transcriptional landscape of porcine cardiac and skeletal muscles reveals differences of aging. \%J Oncotarget. 9(2)

Jieping, H., Z. Qiuzhi, W. Shuzhe, W. Xuefeng, L. Fen, and M. Yun. 2019. High-Throughput RNA Sequencing Reveals NDUFC2 -AS IncRNA Promotes Adipogenic Differentiation in Chinese Buffalo ( Bubalus bubalis L). \%J Genes. 10(9)

Kavitha, M., and S. Shankar. 2020. Skeletal muscle: A review of molecular structure and function, in health and disease. \%J Wiley interdisciplinary reviews. Systems biology and medicine. 12(1)

Kyun, K. H., L. Y. Sun, S. Umasundari, M. Ankit, and D. Anindya. 2006. Muscle-specific microRNA miR-206 promotes muscle differentiation. \%J The Journal of cell biology. 174(5)

Lu, C., J. Zhang, S. He, C. Wan, A. Shan, Y. Wang, L. Yu, G. Liu, K. Chen, J. Shi, Y. Zhang, and R. Ni. 2013. Increased a-tubulin $1 b$ expression indicates poor prognosis and resistance to chemotherapy in hepatocellular carcinoma. Digestive diseases and sciences 58(9):2713-2720. doi: 10.1007/s10620-0132692-z

Lukiw, W. J. 2013. Circular RNA (circRNA) in Alzheimer's disease (AD). Frontiers in genetics 4:307. doi: 10.3389/fgene.2013.00307

M, V. B., and P. R. Jeroen. 2017. Commentary: FUS affects circular RNA expression in murine embryonic stem cell-derived motor neurons. \%J Frontiers in molecular neuroscience. 10

Manuel, H.-H. J., G.-G. E. G, B. C. E, and R. M. A. 2017. The myogenic regulatory factors, determinants of muscle development, cell identity and regeneration. \%J Seminars in cell \& developmental biology. 72 
Margaret, B. 2006. Myogenic progenitor cells and skeletal myogenesis in vertebrates. \%J Current opinion in genetics \& development. $16(5)$

Margaret, B., B. Lola, C. Ted, D. Philippe, H. Juliette, M. Sigolène, M. Didier, R. Didier, and R. Frédéric. 2003. The formation of skeletal muscle: from somite to limb. \% J Journal of anatomy. 202(1)

P, B., H. T, and L. A. B. 2001. The origin of skeletal muscle stem cells in the embryo and the adult. \%J Current opinion in cell biology. 13(6)

Reut, A.-F., M. Markus, P. N. Reddy, I. Andranik, B. Osnat, H. Mor, E. Naveh, M. Sebastian, R. Nikolaus, and K. Sebastian. 2014. circRNA biogenesis competes with pre-mRNA splicing. \%J Molecular cell. 56(1)

Sanger, H. L., G. Klotz, D. Riesner, H. J. Gross, and A. K. Kleinschmidt. 1976. Viroids are single-stranded covalently closed circular RNA molecules existing as highly base-paired rod-like structures. Proceedings of the National Academy of Sciences of the United States of America 73(11):3852-3856. doi: 10.1073/pnas.73.11.3852

Sebastian, M., J. Marvin, E. Antigoni, T. Francesca, K. Janna, R. Agnieszka, M. Luisa, M. S. D, G. L. H, M. Mathias, L. Alexander, Z. Ulrike, L. Markus, K. Christine, I. N. Ferdinand, and R. Nikolaus. 2013. Circular RNAs are a large class of animal RNAs with regulatory potency. \%J Nature. 495(7441)

Simona, G., C. Beatrice, F. Germana, and M. Fabio. 2018. Circular RNAs in Muscle Function and Disease. International journal of molecular sciences 19(11)

T, H. M., and C.-P. M. 1979. Electron microscopic evidence for the circular form of RNA in the cytoplasm of eukaryotic cells. \%J Nature. 280(5720)

Tasneem, K., M. R. Sondekoppa, K. Avak, S. Nahum, and B. Juergen. 2006. Poly(A)-binding protein binds to A-rich sequences via RNA-binding domains $1+2$ and 3+4. \%J RNA biology. 3(4)

Thomas, B., and G. Mathias. 2011. Transcriptional mechanisms regulating skeletal muscle differentiation, growth and homeostasis. \%J Nature reviews. Molecular cell biology. 12(6)

Wei, L., F. Tingting, F. Xing, Y. You, Y. Junjie, Z. Zhen-Ao, L. Junwei, S. Zhenya, D. Wenbo, and H. Shijun. 2018. Signature of circular RNAs in human induced pluripotent stem cells and derived cardiomyocytes. \%J Stem cell research \& therapy. 9(1)

Xuefeng, W., L. Hui, Y. Jiameng, H. Dan, D. Dong, H. Yongzhen, L. Xianyong, P. Martin, L. Chuzhao, L. Fengpeng, B. Yueyu, and C. Hong. 2017. Circular RNA profiling reveals an abundant circLMO7 that regulates myoblasts differentiation and survival by sponging miR-378a-3p. \%J Cell death \& disease. $8(10)$

Yang, Z., Z. Xiao-Ou, C. Tian, X. Jian-Feng, Y. Qing-Fei, X. Yu-Hang, Z. Shanshan, Y. Li, and C. Ling-Ling. 2013. Circular intronic long noncoding RNAs. \%J Molecular cell. 51(6) 
Yuying, L., C. Xiaona, S. Hao, and W. Huating. 2018. Long non-coding RNAs in the regulation of skeletal myogenesis and muscle diseases. Cancer letters 417

Zhaoyong, L., H. Chuan, B. Chun, C. Liang, L. Mei, W. Xiaolin, Z. Guolin, Y. Bin, H. Wanchen, D. Limin, Z. Pengfei, C. Zhaoxia, W. Qingfa, Z. Yi, J. Ya, X. Ping, L. Huijie, and S. Ge. 2015. Exon-intron circular RNAs regulate transcription in the nucleus. \%J Nature structural \& molecular biology. 22(3)

\section{Tables}

Table 1 Summary of reads mapping to the buffalo reference genome

\begin{tabular}{|l|c|c|c|c|c|c|}
\hline $\begin{array}{l}\text { Samples } \\
\text { Embryo }\end{array}$ & $\begin{array}{c}\text { Proliferation } \\
1\end{array}$ & $\begin{array}{c}\text { Proliferation } \\
\text { 2 }\end{array}$ & Proliferation 3 & $\begin{array}{c}\text { Differentiation } \\
\text { A }\end{array}$ & $\begin{array}{c}\text { Differentiation } \\
\text { B }\end{array}$ & $\begin{array}{c}\text { Differentiation } \\
\text { C }\end{array}$ \\
\hline Raw reads & $104,672,134$ & $108,274,434$ & $108,331,154$ & $106,828,636$ & $104,100,068$ & $103,416,606$ \\
\hline Clean reads & $100,061,338$ & $103,546,858$ & $102,399,234$ & $101,952,364$ & $96,266,686$ & $98,333,562$ \\
\hline $\begin{array}{l}\text { Mapped } \\
\text { reads }\end{array}$ & $100,039,479$ & $103,527,375$ & $103,527,375$ & $101,936,644$ & $96,227,208$ & $98,317,629$ \\
\hline $\begin{array}{l}\text { Mapping } \\
\text { Ratio }\end{array}$ & $99.98 \%$ & $99.98 \%$ & $99.97 \%$ & $99.98 \%$ & $99.96 \%$ & $99.98 \%$ \\
\hline $\begin{array}{l}\text { Uniquely } \\
\text { mapped } \\
\text { reads }\end{array}$ & $77,493,289$ & $78,489,074$ & $77,045,729$ & $81,224,612$ & $75,896,937$ & $77,379,908$ \\
\hline $\begin{array}{l}\text { Unique } \\
\text { mapping } \\
\text { Ratio }\end{array}$ & $77.46 \%$ & $75.81 \%$ & $75.26 \%$ & $79.68 \%$ & $78.87 \%$ & $78.70 \%$ \\
\hline
\end{tabular}

Table 2 Results from the assembly of circRNAs (including flanking introns lengths)

\begin{tabular}{|l|c|c|c|c|c|c|}
\hline Item & CircRNA & Min. length & Mean length & Median length & Max. length & Total length \\
\hline Number & 3,142 & 182 & $17,474.44$ & $9,725.5$ & 189,473 & $54,904,696$ \\
\hline
\end{tabular}

Table 3 Differential binding protein of circPICALM obtained by RNA pulldown experiment

\begin{tabular}{|l|c|l|c|c|c|c|}
\hline Accession & Gene & \multicolumn{1}{|c|}{ Description } & circPICALM & control & $\begin{array}{l}\text { log2(Mean } \\
\text { SP) }\end{array}$ & $\begin{array}{l}\text { Diff } \\
\text { Sig }\end{array}$ \\
\hline F1MTV9 & NPM1 & Nucleophosmin & 9 & 1 & 2.322 & ++ \\
\hline P81947 & TUBA1B & Tubulin alpha-1B chain & 11 & 1 & 2.585 & ++ \\
\hline E1BF59 & PLEC & Plectin & 11 & 3 & 2.807 & ++ \\
\hline E1BPW2 & ERC1 & $\begin{array}{l}\text { ELKS/RAB6-interacting/CAST family } \\
\text { member 1 }\end{array}$ & 4 & 1 & 1.322 & + \\
\hline F1MYI2 & HNRNPM & Uncharacterized protein & 4 & 1 & 1.322 & + \\
\hline A4FUD0 & MTHFD1 & MTHFD1 protein & 6 & 2 & 2 & + \\
\hline Q3MHY1 & CSRP1 & Cysteine and glycine-rich protein 1 & 8 & 3 & 2.459 & + \\
\hline E1BFB0 & SPTAN1 & Spectrin alpha, non-erythrocytic 1 & 37 & 16 & 4.7279 & + \\
\hline A2VDN8 & CORO1C & Coronin & 15 & 7 & 3.459 & + \\
\hline Q6Q137 & SEPT & Septin-7 & 8 & 3 & 2.459 & + \\
\hline F1N6H4 & MACF1 & Uncharacterized protein & 8 & 4 & 2.585 & + \\
\hline Q29RK2 & PC & Pyruvate carboxylase, mitochondrial & 13 & 6 & 3.247 & + \\
\hline F1MYC9 & SPTBN1 & Spectrin beta chain & 46 & 24 & 5.1293 & + \\
\hline
\end{tabular}




\section{Supplemental File Legends}

Figure S1. Competing endogenous RNA network in buffalo myoblasts.

Additonal file 1. A total of 3,142 candidates were expressed in proliferated and differentiated buffalo myoblasts.

Additonal file 2. Differentially expressed circRNAs identified in buffalo myoblasts.

Additonal file 3. Gene Ontology enrichments and Kyoto Encyclopedia of Genes and Genomes pathway enrichment assays.

Additonal file 4. The host gene of differentially expressed circRNAs identified in buffalo myoblasts.

Table S1. Primers for quantitative real time PCR and vector construction.

Text S1. Sequence of buffalo circPICALM.

\section{Figures}


a

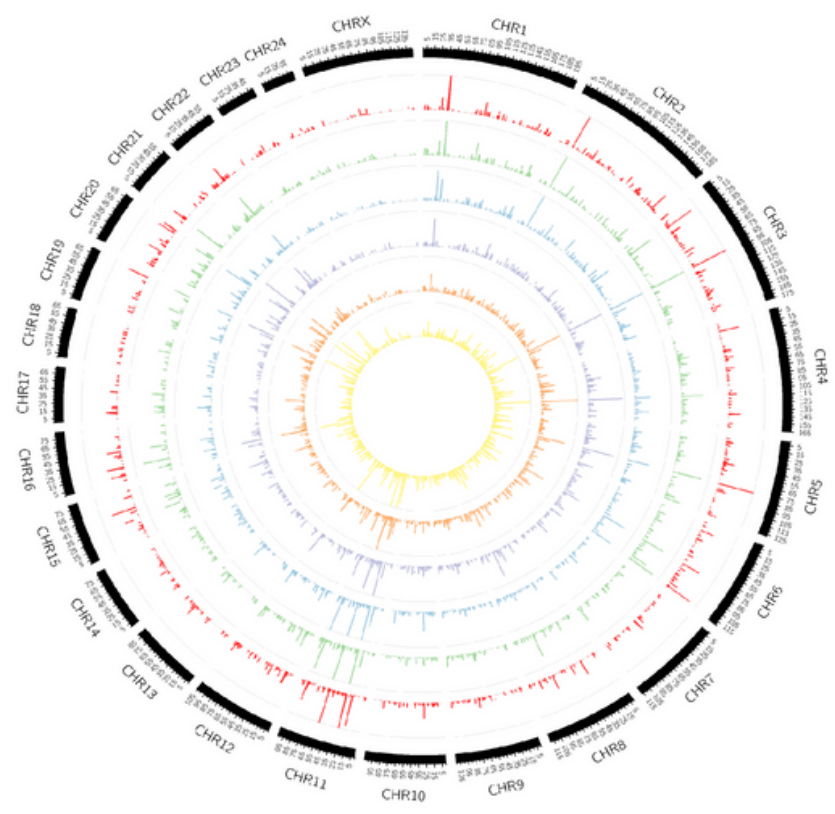

$\mathrm{c}$

Proliferating phase

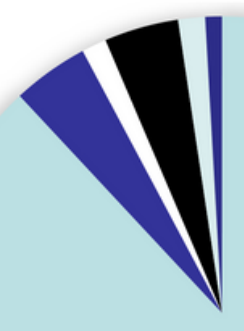

InLASIC $(88 \%)$

b

\section{Proliferating phase Differentiation phase}

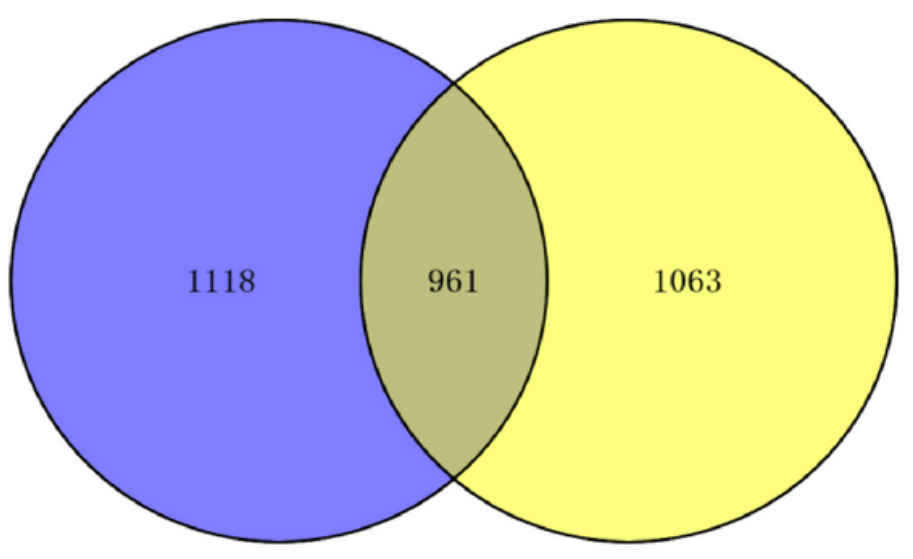

- ALTER_EXON(4\%)

INTRON $(1 \%)$

- OVERLAP_EXON(4\%)

ANTISENSE( $2 \%$ )

- INTERGENIC(1\%)
Differentiation phase

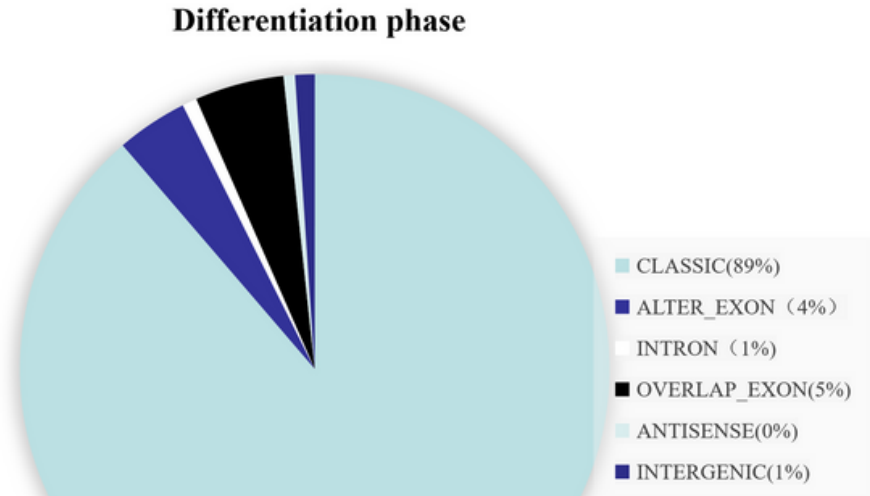

\section{Figure 1}

Expression profiles of circRNAs in proliferating and differentiating buffalo myoblasts. (a) Circos plot showing the distribution of circRNA in different chromosomes. (b) The Venn diagrams of different circRNA found at two growth stages (proliferation and differentiation) of buffalo myoblasts. (c) The origin of circRNA described in this study in the buffalo genome. 
a

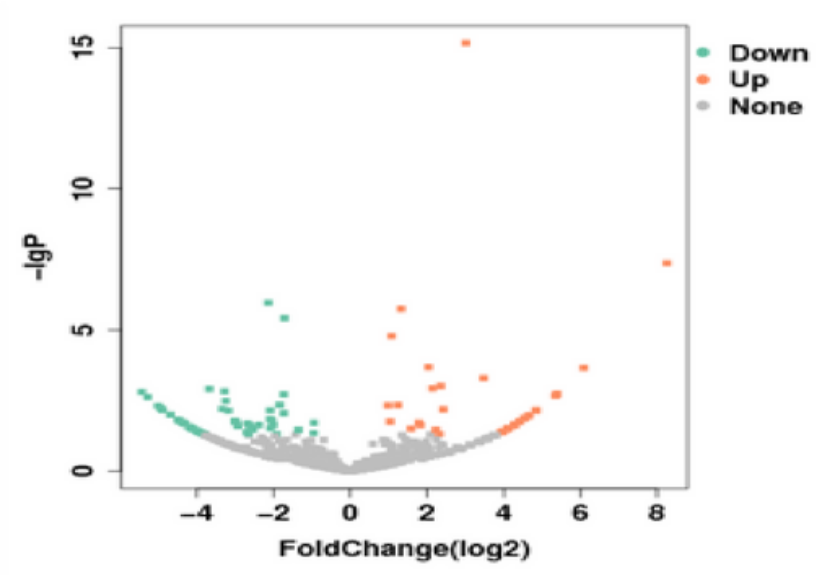

c

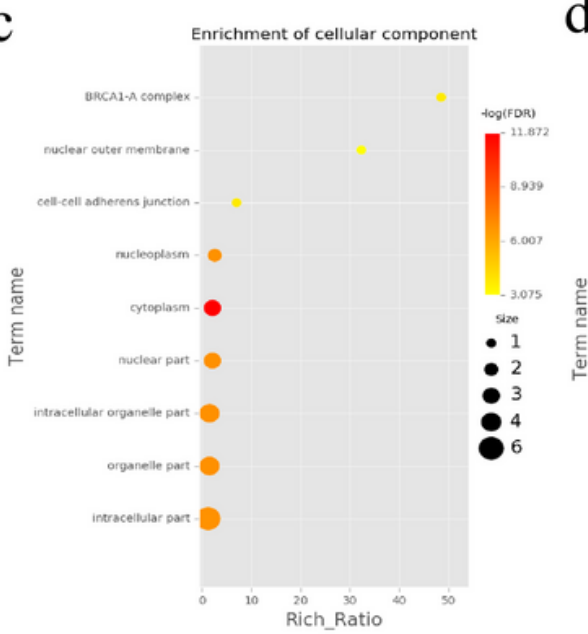

f

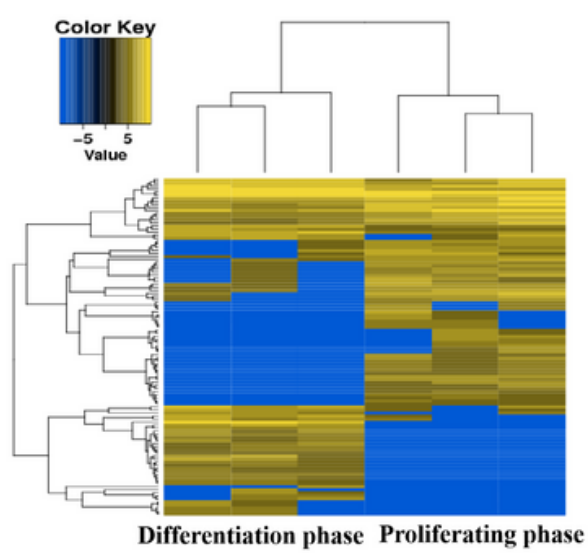

b

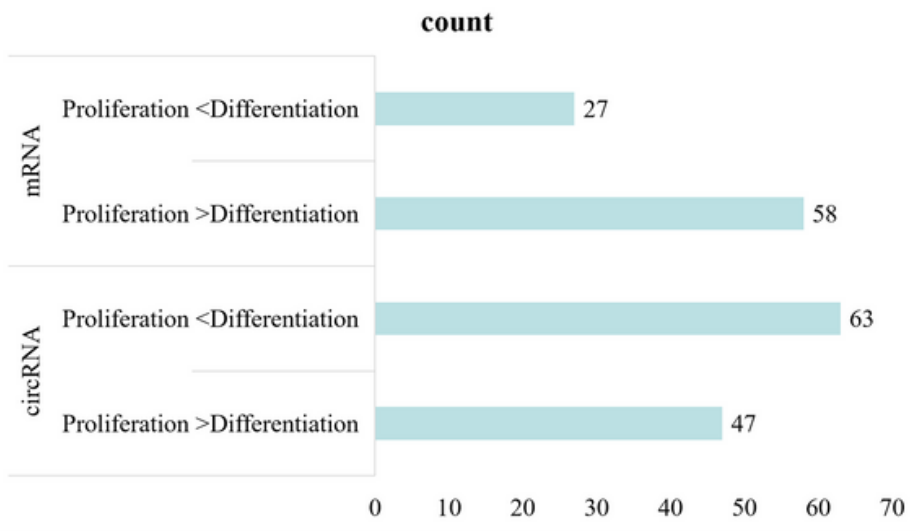

e

Enrichment of biological process

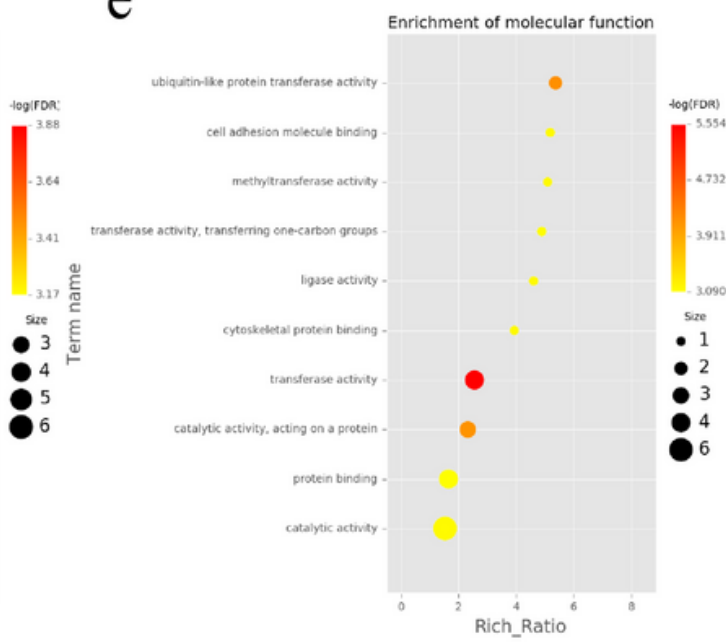

g

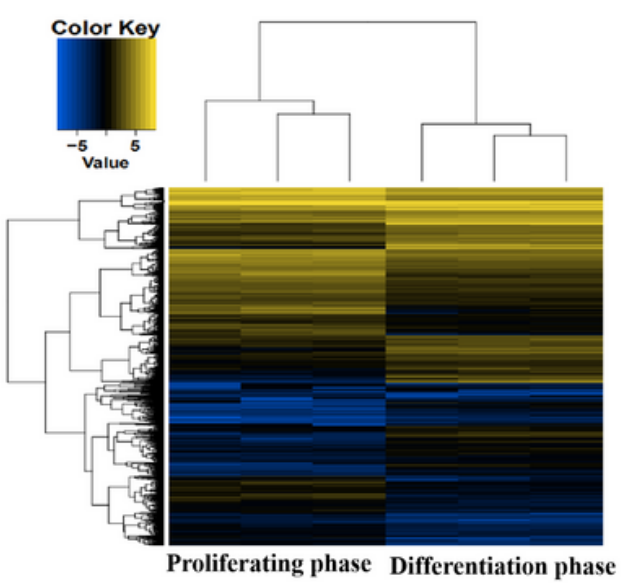

Figure 2

Differentially expressed circular RNAs in buffalo muscle tissues. (a) Differentially expressed circRNA volcano map. (b) Expression differences of circRNA and linear mRNA in two developmental stages of buffalo muscle. (c-e) GO entries that describe the molecular function of genes, cell components, q enrichment of biological processes, and the degree of enrichment. (f) Cluster analysis of circRNA differentially expressed in buffalo myoblasts during proliferation and differentiation. (g) Cluster heat 
maps showing the abundance of corresponding linear host transcripts of circRNA with the greatest difference between proliferation and differentiation phases.

a

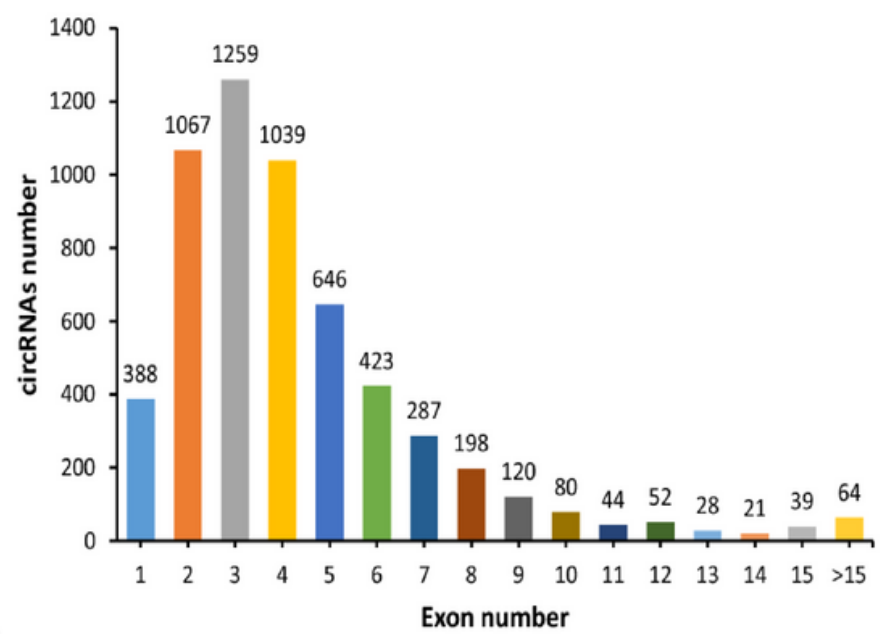

c

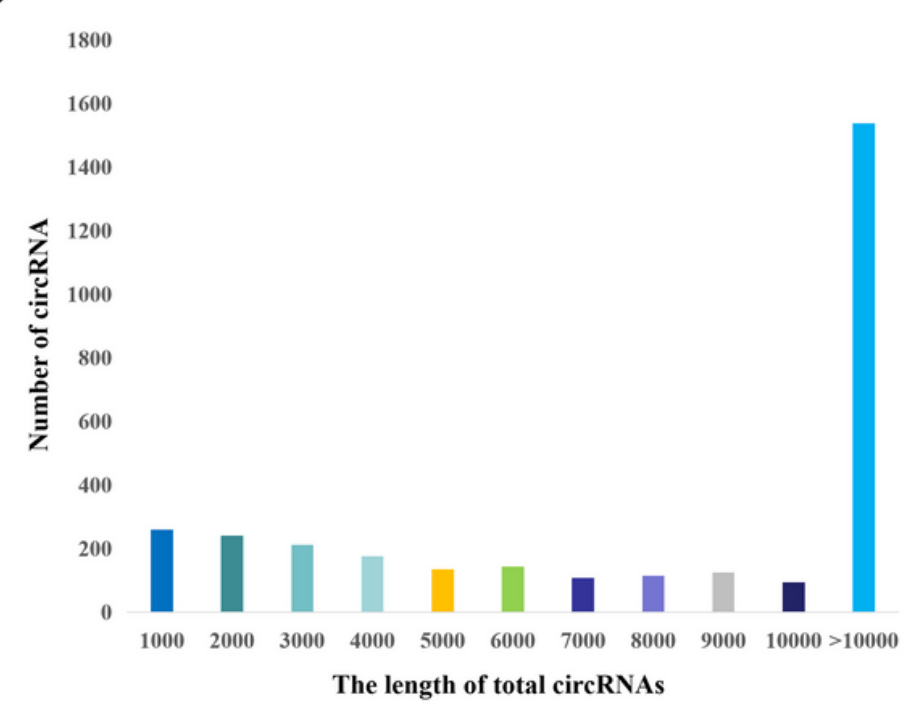

b

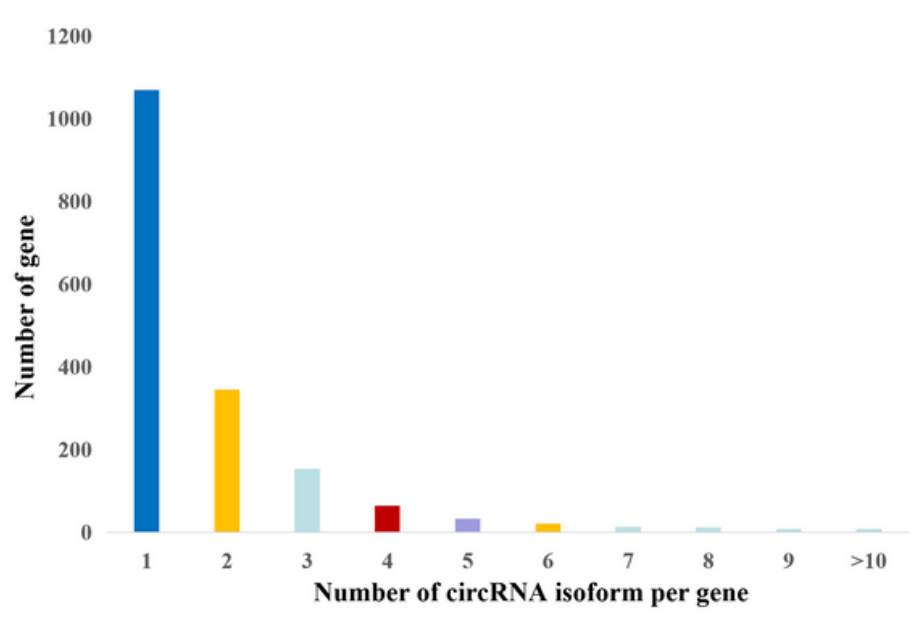

d

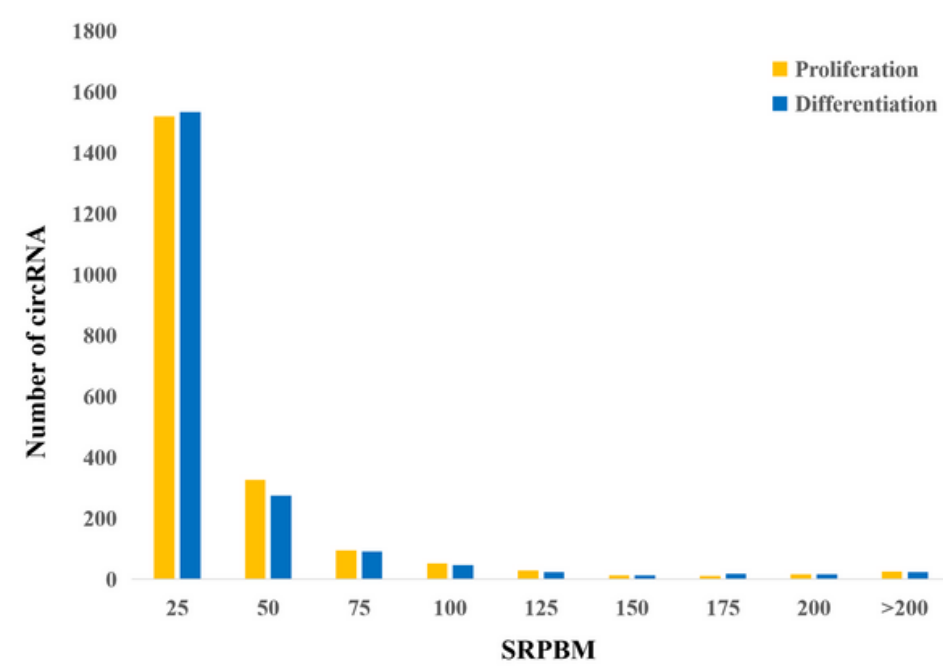

\section{Figure 3}

Characteristics of circular RNA in buffalo myoblasts. (a) Number of circRNAs contained different number of exons. (b) Number of circRNAs produced from one gene. (c) Size distribution of the circRNAs including flanking introns lengths. (d) The overall expression distribution of circRNA during proliferation and differentiation. 
a

b

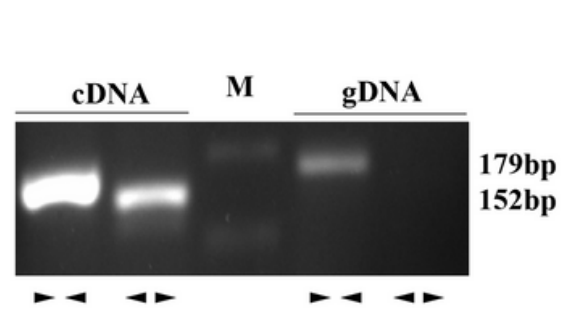

$\mathrm{d}$

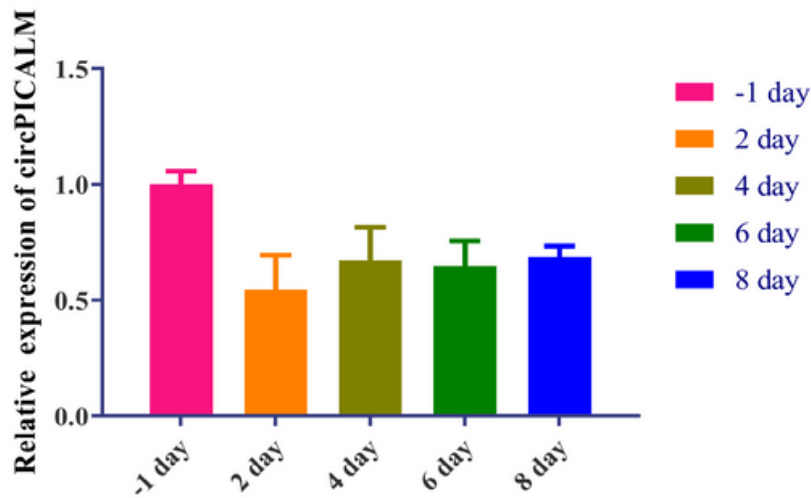

C

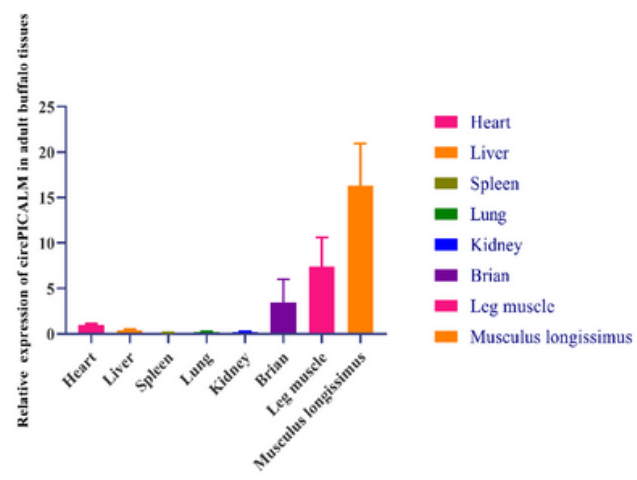

e

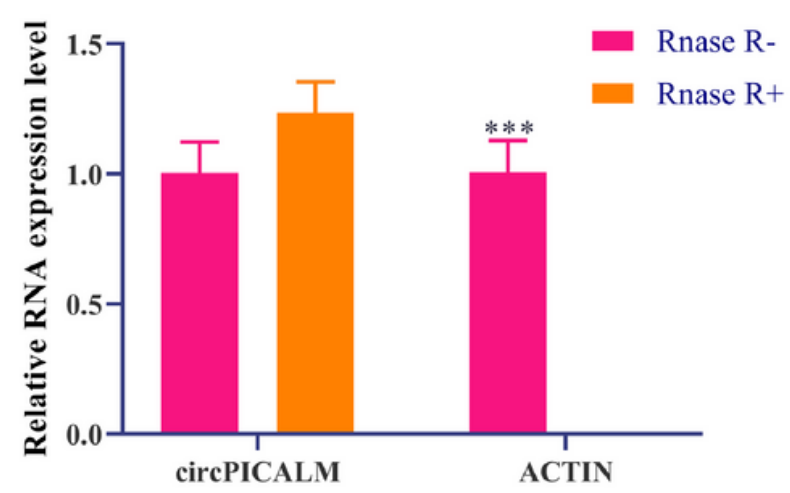

Figure 4

The expression of circPICALM. (a) Using cDNA and genomic DNA of buffalo as templates, and PCR amplification was performed in junction region of circPICALM to verify its authenticity. (b) CircPICALM was differentially expressed in different fetal buffalo tissues. (c) CircPICALM was differentially expressed in different adult buffalo tissues. (d) CircPICALM was differentially expressed in buffalo myoblasts in different days. (e) The authenticity of circPICALM was verified by RNase R digestion. 
a

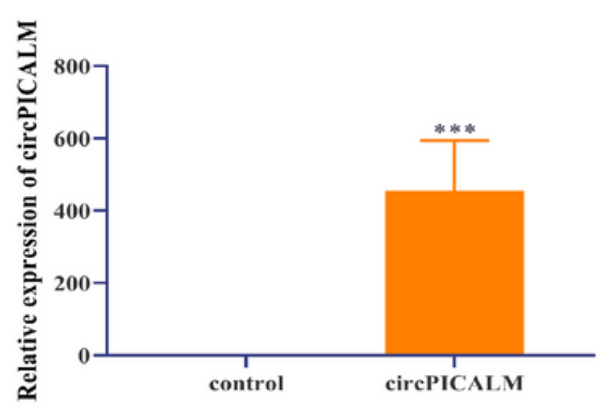

control

- circPICALM

$\mathrm{C}$
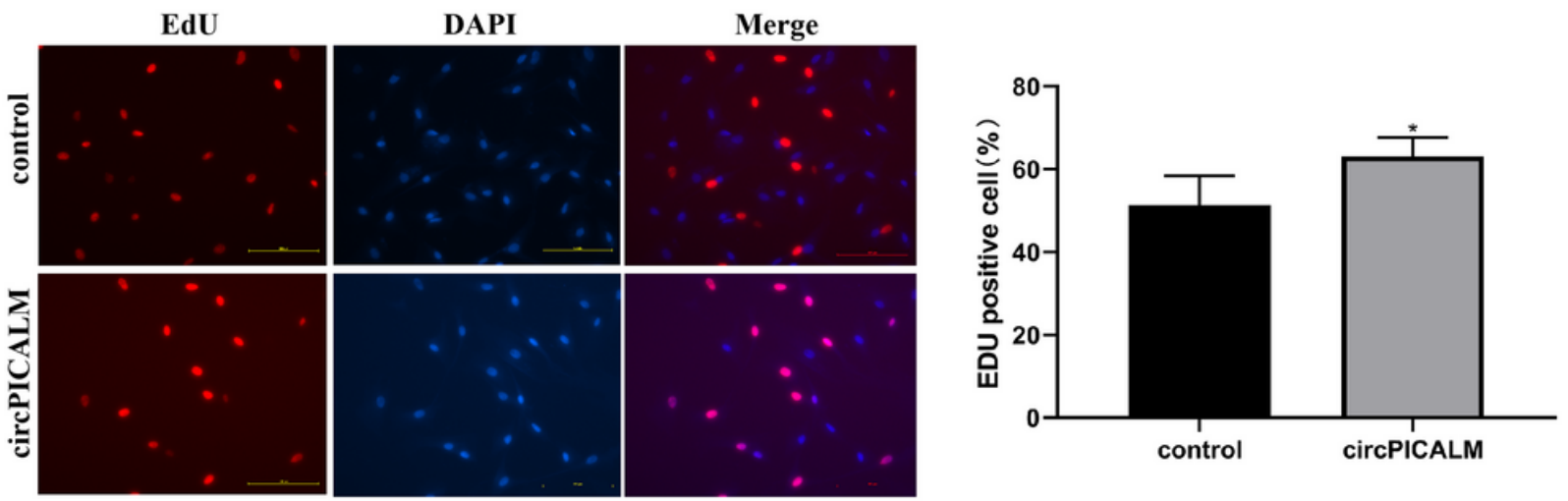

d
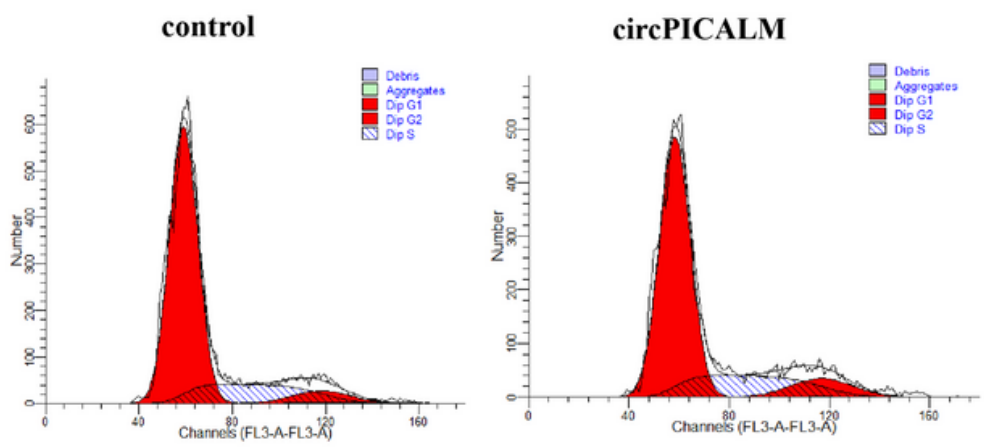

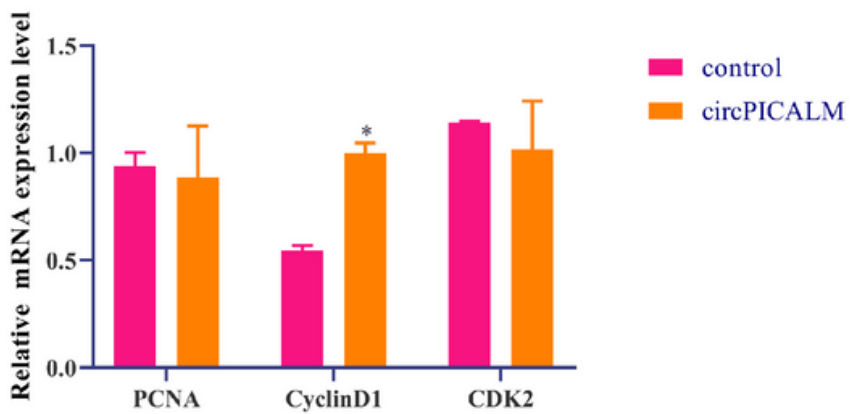

control

口 cirCPICALM

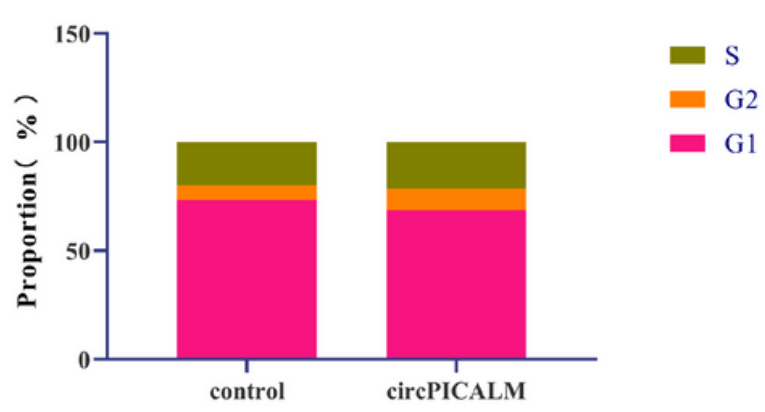

Figure 5

Effects of circPICALM on myoblasts proliferation. (a) circPICALM overexpression level. (b) qRT-PCR to detect the mRNA expressions of muscle proliferating genes PCNA, CyclinD1 and CDK2. (c) The EdU assay was used to detect the influence of circPICALM on cell proliferation. (d) The flow cytometry to analyze the influence of circPICALM on cell cycle. 
a

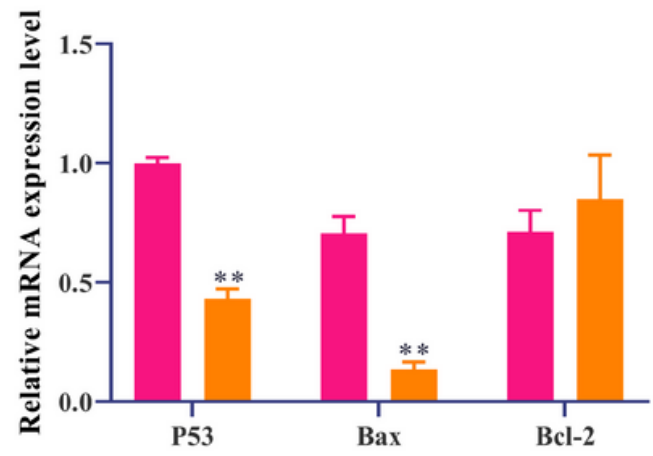

C

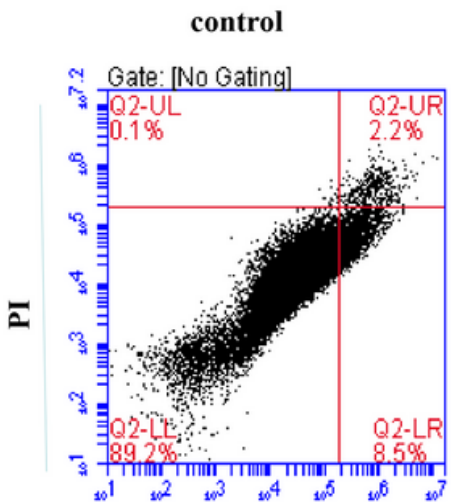

b

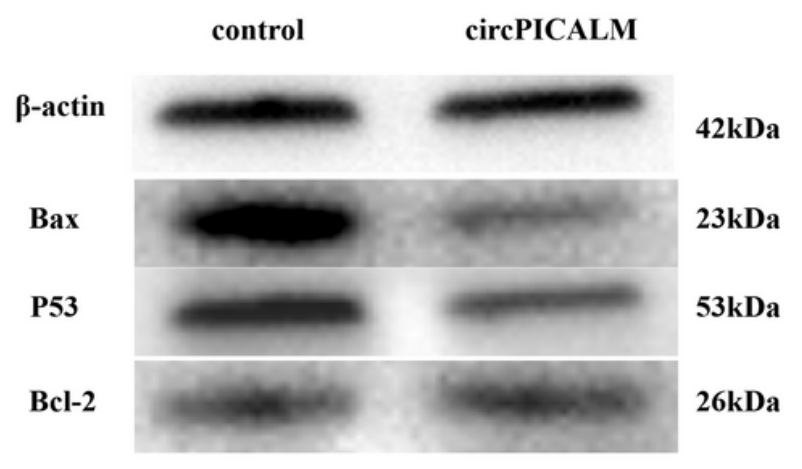

circPICALM
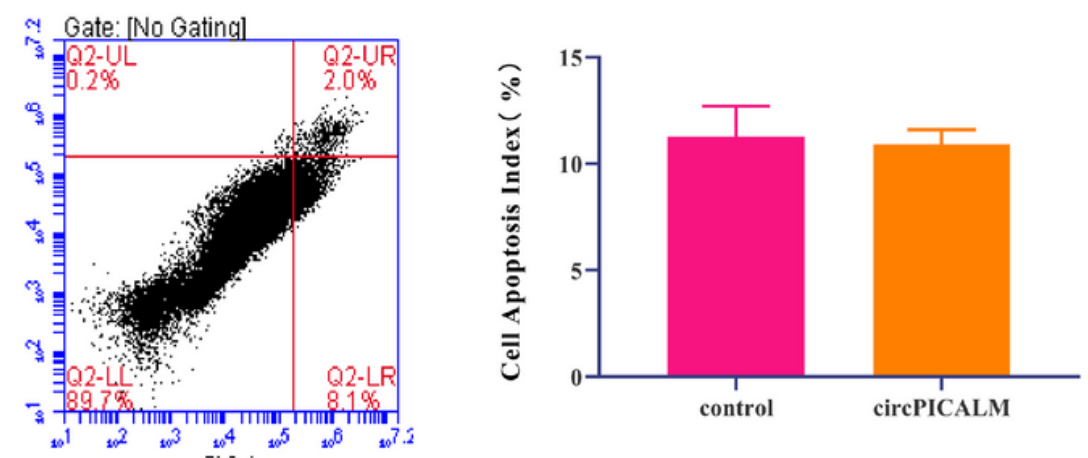

control

circPICALM

Annexin V-FITC

\section{Figure 6}

CircPICALM inhibited the apoptosis of buffalo myoblasts. (a) qPCR to detect the mRNA expressions of apoptosis genes P53, Bax and Bcl-2. (b) Western blot to detect P53, Bax and Bcl-2 proteins. (c) Flow cytometry to detect apoptosis. 

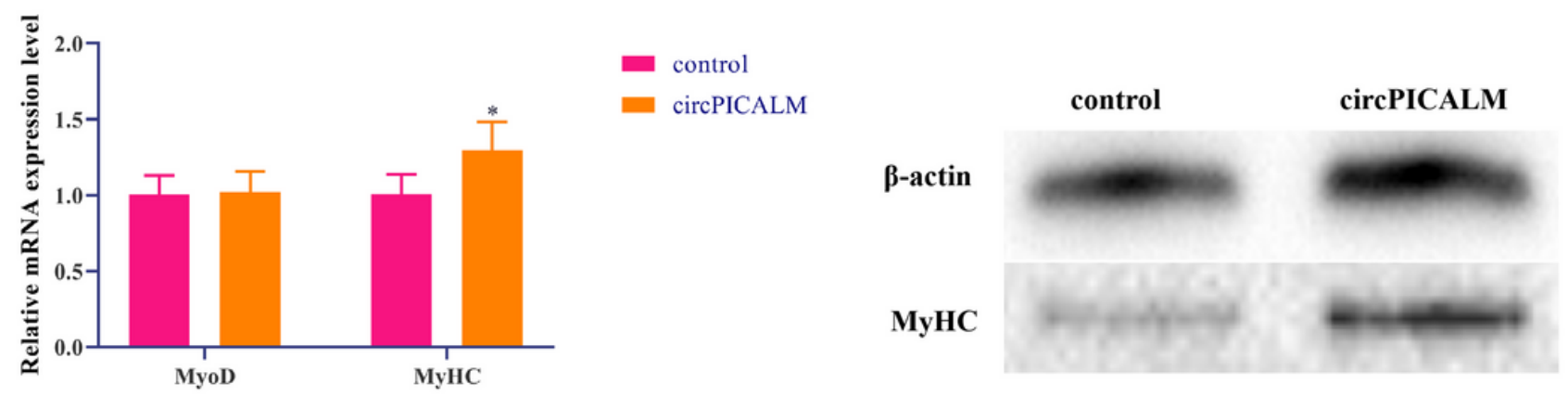

c

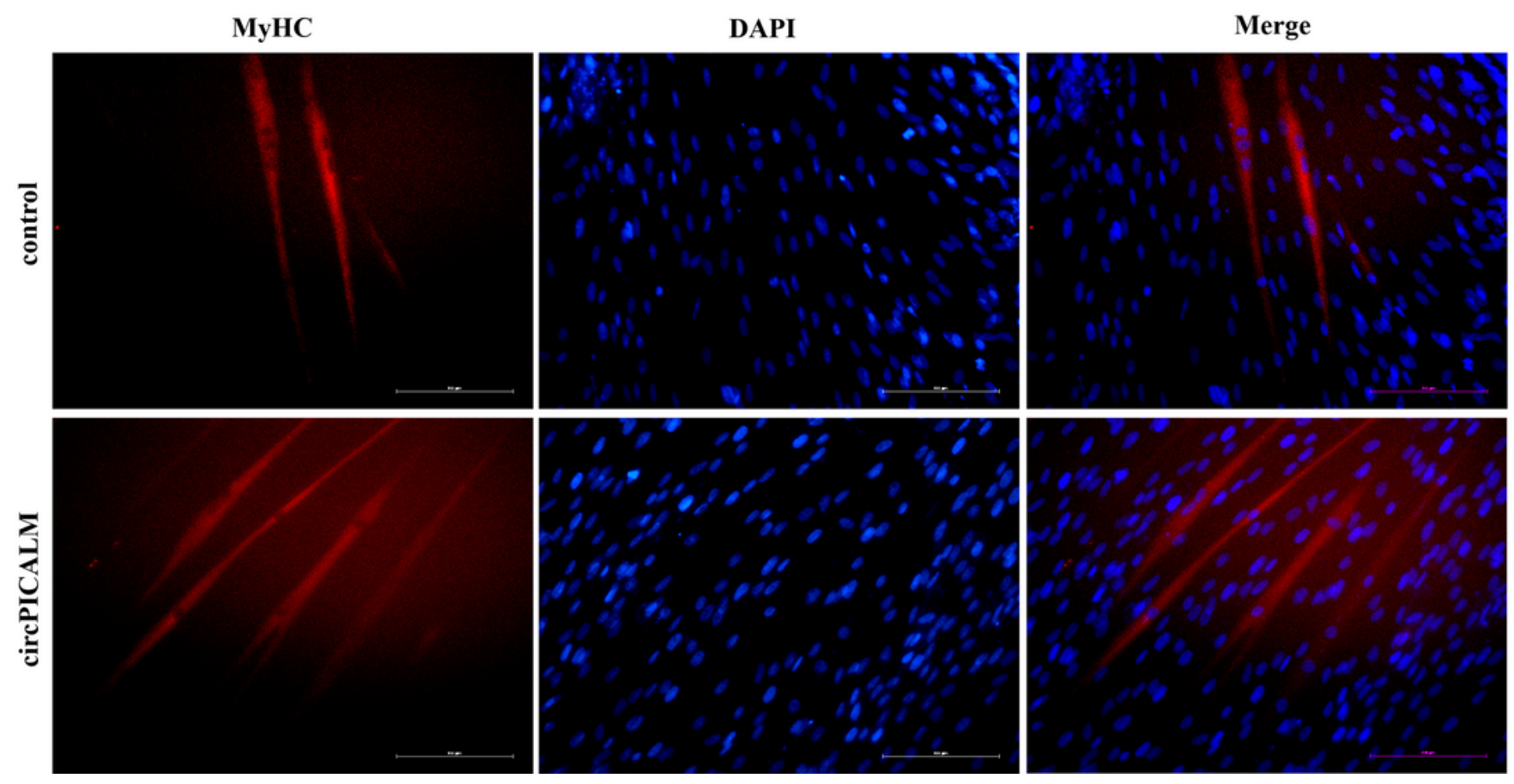

\section{Figure 7}

CircPICALM can promote buffalo myoblast differentiation. (a) qPCR to detect the mRNA expressions of MyoD and MyHC muscle genes. (b) Western blot to detect MyHC protein translation. (c) The effect of circPICALM transfection on MyHC gene expression in buffalo myoblasts during differentiation was detected by immunofluorescence assay.

2

\section{Figure 8}

CircPICALM promotes skeletal muscle regeneration. (a) Expression level after 3 days of circPICALM plasmid injection for skeletal muscle injury. (b) qPCR detecting mRNA levels of myogene-related genes 3 days after transfection with tibialis anterior by circPICALM expression plasmid. (c) Expression level after 7 days of circPICALM plasmid injection for skeletal muscle injury. (d) qPCR detecting mRNA levels of 
myogenesis-related genes 7 days after transfection with tibialis anterior by circPICALM expression plasmid. (e) HE-staining CTX-injected muscle after transfection circPICALM expression plasmid.

a

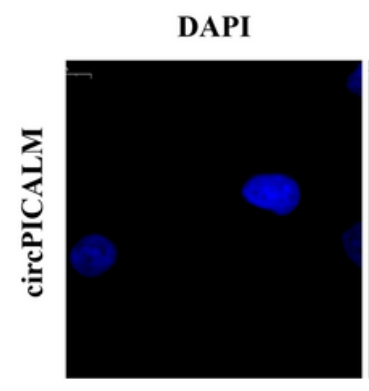

$\mathrm{b}$

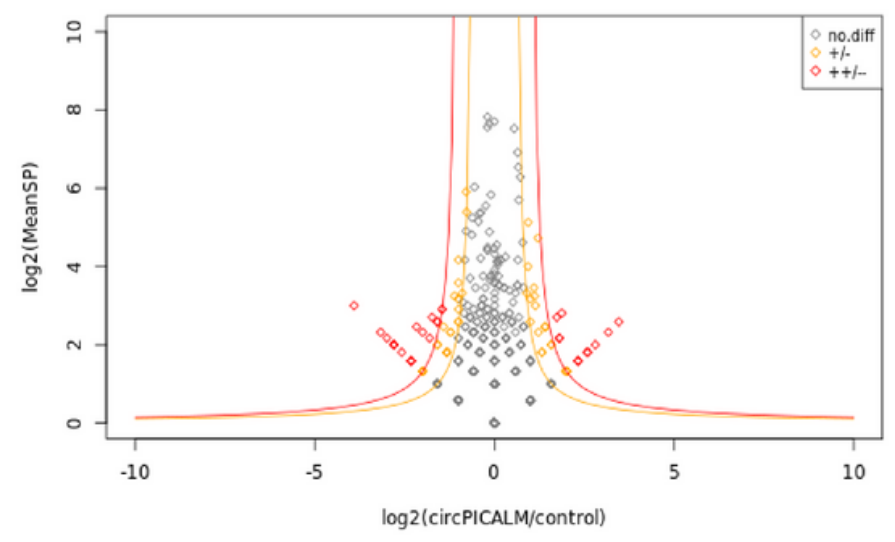

Figure 9

The subcellular localization and binding proteins of circPICALM. (a) CircPICALM was subcellular localized with RNA-FISH. (b) CircPICALM RNA-pulldown differential protein distribution.

\section{Supplementary Files}

This is a list of supplementary files associated with this preprint. Click to download.

- TextS1.docx

- Additionalfile3.xls

- Additionalfile1.xls

- FigureS1.tif

- Additionalfile4.xls

- TableS1.docx

- Additionalfile2.xls 